\title{
Results of Catheter Ablation of Ventricular Tachycardia Using Direct Current Shocks
}

\author{
FRED MORADY, MELVIN M. SCHEINMAN, JERRY C. GRIFFIN, \\ JOHN M. HERRE, and WILLIAM H. KOU \\ From the Divisions of Cardiology, Department of Internal Medicine, Moffitt Hospital, San \\ Francisco, California and the University of Michigan Medical Center, Ann Arbor, Michigan
}

\begin{abstract}
MORADY, F., ET AL.: Results of Catheter Ablation of Ventricular Tachycardia Using Direct Current Shocks. Thirty-three patients with recurrent ventricular tachycardia (VT) underwent catheter ablation with direct-current shocks. One to four shocks of 100-300 joules were delivered to the presumed VT exit sites as identified by endocardial mapping and pace mapping. Fifteen patients (45\%) had no recurrence of symptomatic VT during a follow-up interval of $15.5 \pm 10$ months (mean \pm standard deviation). Five patients experienced six nonfatal complications (new VT or ventricular fibrillation, transient neurological deficit, atrioventricular block, brachial artery thrombosis). In conclusion, catheter ablation in selected patients with recurrent VT has the potential for preventing recurrences of VT over the long-term and is relatively safe. (PACE, Vol. 12, January Part II 1989)
\end{abstract}

ventricular tachycardia, endocardial mapping, catheter ablation

\section{Introduction}

Transcatheter direct current shocks delivered to the endocardium of the right or left ventricle have at times been found to be effective in preventing recurrences of ventricular tachycardia (VT). The purpose of this report is to summarize the results of attempted catheter ablation of VT in 33 patients.

\section{Methods}

The subects of this report consist of 33 consecutive patients who underwent an attempt at catheter ablation of VT at either Moffitt Hospital, San Francisco or the University of Michigan Medical Center between September 1982 and June 1986. Selection criteria included a history of recurrent, symptomatic, sustained VT and either spontaneous or induced VT in the electrophysiology laboratory which was hemodynamically

Address for reprints: Fred Morady, M.D., Division of Cardiology, University Hospital, 1500 East Medical Center Drive, Bl F245-0022, Ann Arbor, MI 48109-0022. stable and which could be mapped. In most cases, patients had only a single configuration of VT.

There were 32 men and one woman, and their mean age was $56 \pm 14$ years $( \pm$ standard deviation). The underlying heart disease consisted of coronary artery disease in 22 patients, idiopathic dilated cardiomyopathy in two patients, arrhythmogenic right ventricular dysplasia in two patients, and tetralogy of Fallot that had undergone surgical repair in two patients. No structural heart disease was present in five patients. The mean left ventricular ejection fraction was $0.34 \pm 0.17$. Nineteen of the 22 patients who had coronary artery disease had a ventricular aneurysm.

All patients had a history of symptomatic VT, and the most severe symptom resulting from VT consisted of a cardiac arrest in ten patients, syncope in five patients, near syncope in 12 patients, and palpitations and/or chest pain or dyspnea in six patients. Thirteen patients had a history of more than 25 episodes of VT, and 20 patients had a history of between two and 25 episodes. In eight patients the VT was incessant for 3 to 7 days before the attempt at catheter ablation. All patients 
had been treated unsuccessfully with at least two Class I antiarrhythmic drugs. Nineteen patients were refractory to amiodarone. The mean number of total drug trials that were ineffective in controlling VT before catheter ablation was 3.7 \pm 1.2 .

\section{Electrophysiological Testing}

Patients were studied in the fasting, unsedated state after they provided informed consent. Antiarrhythmic drug therapy was discontinued before the procedure in 18 patients and was continued in 15 patients who had had a partial response to antiarrhythmic drug therapy. Two 6 French quadripolar electrode catheters were inserted into a femoral vein and positioned against the right ventricular apex and outflow tract or septum. A 6 French quadripolar electrode catheter was inserted through a femoral or brachial artery and positioned within the left ventricle. Heparin was administered intravenously at an initial dose of 3,000 units followed by 1,000 units every hour. Electrocardiographic leads $\mathrm{V}_{1}$, I and III, the intracardiac electrograms, and a continuous recording of the arterial pressure were displayed on an oscilloscope and recorded at a paper speed of 100 to $150 \mathrm{~mm} / \mathrm{s}$. Programmed stimulation was performed with a programmable stimulator. Whenever sustained VT that was hemodynamically stable was induced, a 12-lead electrocardiographic recording was obtained.

Programmed ventricular stimulation was performed with single, double and triple ventricular extrastimuli at two right ventricular sites using basic drive cycle lengths of 600,500 , or 400 ms. Sustained VT was defined as VT more than 30 seconds in duration or requiring direct-current countershock for termination. Nonsustained VT was defined as VT that was six complexes to 30 seconds in duration.

\section{Mapping Technique}

In each patient at least ten ventricular sites were mapped. Whenever multiple morphologies of VT were induced, the VT morphology that had occurred spontaneously was mapped. The endocardial activation time at each mapping site was defined as the interval between the first rapid de- flection of the intracardiac electrogram and the earliest deflection of the QRS complex in leads I, III, or $\mathrm{V}_{1}$ during VT.

Pace mapping was performed by pacing at a cycle length similar to the VT cycle length. Twelve lead electrocardiograms were recorded during pacing. However, pace mapping could not be accomplished in seven patients because of the inability to obtain ventricular capture with pacing stimuli up to $20 \mathrm{~mA}$ in strength.

Pace maps were considered "excellent" if the QRS complexes were identical or very similar to the QRS complexes during VT in each of the 12 leads (Fig. 1). Pace maps were considered "good" if the QRS complexes had the same bundle branch block configuration and axis as the VT and if the QRS complexes during pacing were very similar to the QRS complexes during VT in ten or 11 of 12 leads. If the QRS complexes during pacing were very similar to the QRS complexes during VT in no more than nine of 12 leads, the pace map was considered "poor".

In the first ten patients in this series, shocks were delivered at sites that had the earliest endocardial activation during VT, without regard to the results of pace mapping. However in subsequent patients shocks were delivered to sites at which endocardial activation preceded the onset of the QRS complex during VT and at which the pace map was either good or excellent.

\section{Catheter Ablation Technique}

The investigational protocol for catheter ablation of VT was approved by the Human Research Committee at the University of California, San Francisco and the University of Michigan. A previously unused 6 French quadripolar electrode catheter (USCI, Billerica, MA, USA) with either 0.5 or $1 \mathrm{~cm}$ between electrodes was positioned against the endocardium at the presumed exit site of the VT. A 16-cm patch electrode (R2 Corporation) was positioned on the chest in the closest possible proximity to the tip of the catheter. The distal electrode of the catheter was connected to the cathodal output of the defibrillator and the patch electrode served as the anode. In patients in whom the exit site of VT was in the intraventricular septum, a catheter was positioned against each side of the septum, such that 


\section{VT, CL $340 \mathrm{~ms}$}
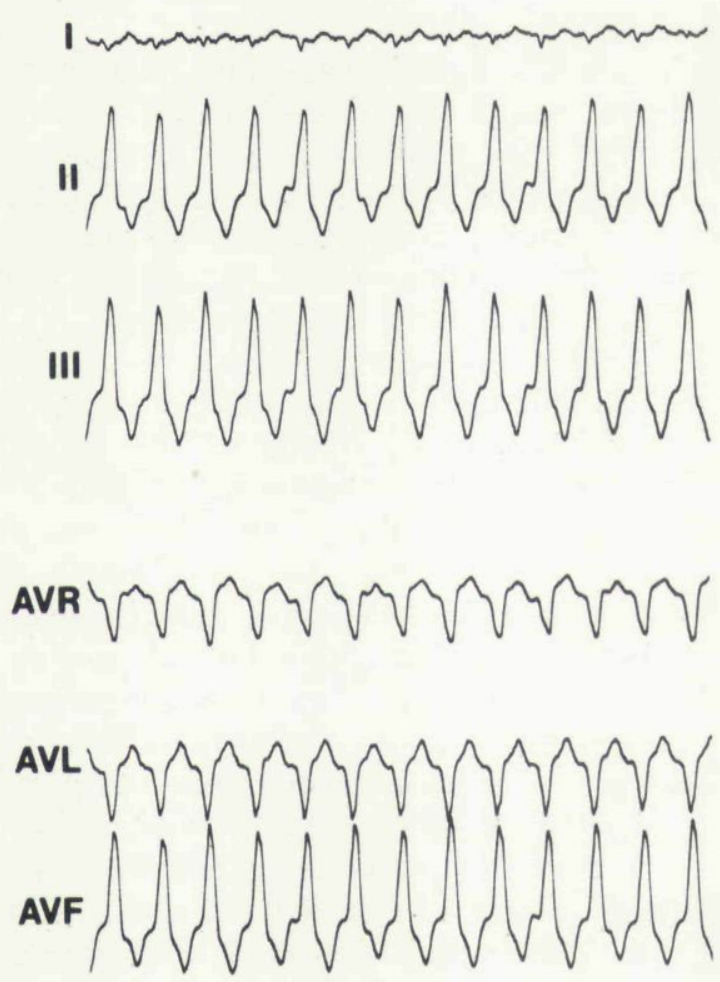

Pacing, CL 300 ms, site 16
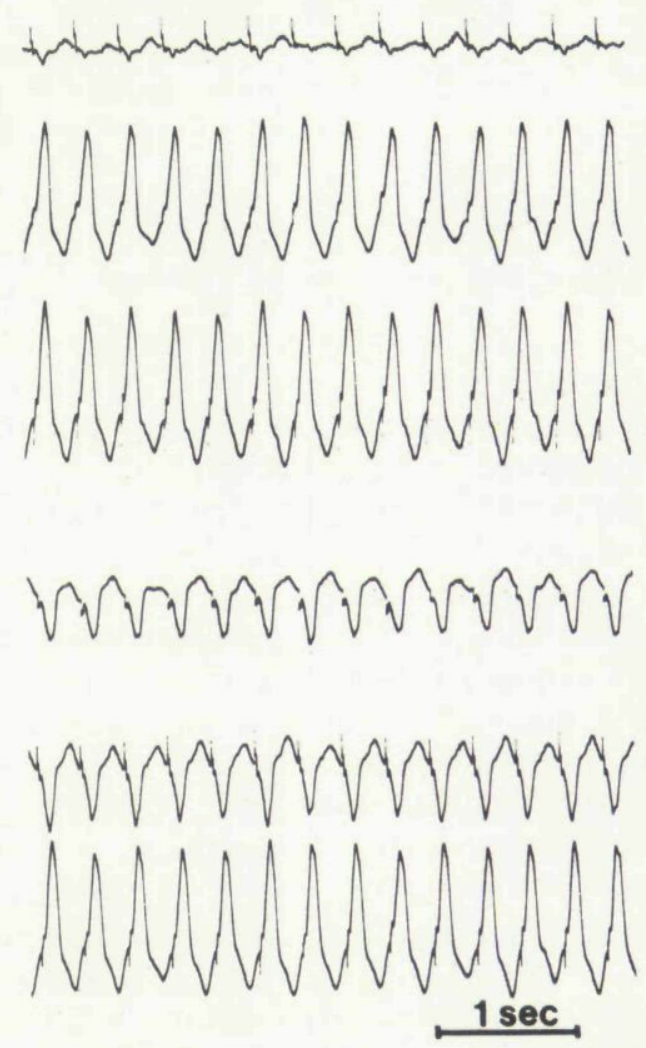

Figure 1A. An example of an "excellent" pace map, with very similar QRS complexes during ventricular tachycardia (VT) and during pacing at the right ventricular outflow tract (site 16) in each of the 12 leads of the electrocardiogram. A. Frontal plane leads

the exit site of the VT was straddled. The distal electrode of the left ventricular catheter served as the cathode and the distal electrode of the catheter on the right ventricular side of the septum served as the anode.

Patients were anesthetized with either intravenous sodium thiopental or methohexitol. Shocks that had a damped sinusoidal configuration and a stored energy of 100 to 300 joules then were delivered in synchronized fashion. Each patient received one to four shocks. Programmed stimulation was performed 30 to 60 minutes after the shocks were delivered to assess whether VT was still inducible. After the procedure, patients were monitored in the hospital and 1 week later, an electrophysiologic study was repeated in patients who had not had inducible VT 30 to 60 minutes after the shocks. If VT was inducible, the patients were treated with antiarrhythmic drugs as guided by the results of electropharmacologic testing.

\section{Follow-Up}

Follow-up information was obtained by interviewing patients either in person or by telephone. A successful outcome of catheter ablation was defined as no recurrence of symptomatic VT during follow-up, either in the absence of antiarrhythmic drug therapy, or while being treated with the same antiarrhythmic drug regimen that had been unsuccessful in controlling VT before the ablation attempt.

\section{Results}

The mean VT cycle length was $412 \pm 91 \mathrm{~ms}$. The results of mapping indicated that the VT exit 


\section{VT, CL $340 \mathrm{~ms}$}
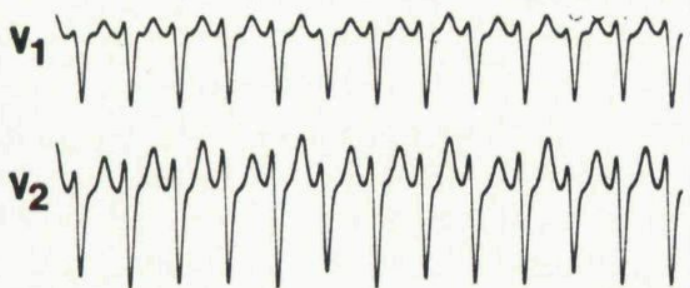

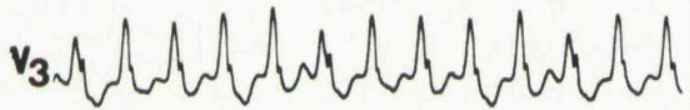
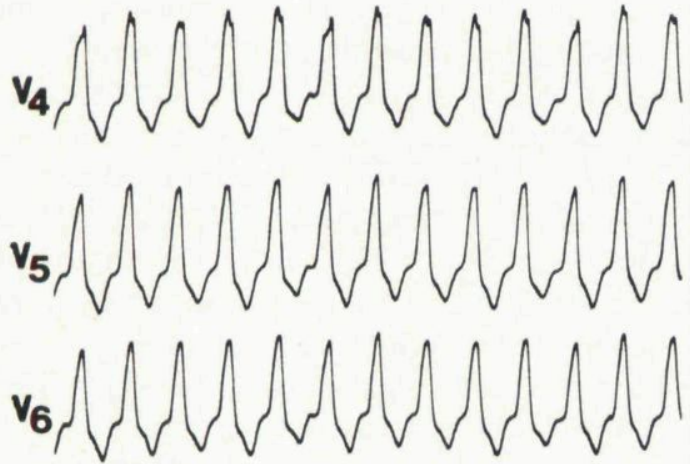

Pacing, CL 300 ms site 16
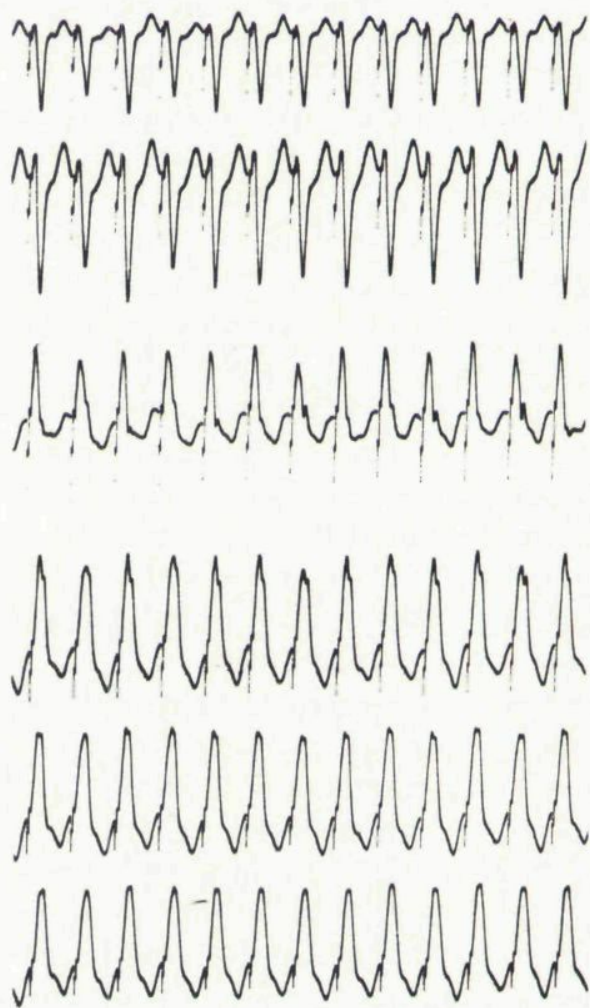

$1 \mathrm{sec}$

Figure 1B. Precordial leads

site was in the left free wall in 13 patients, the interventricular septum in nine patients, and the right ventricle in 11 patients.

In 29 patients, an electrode positioned against the endocardium served as the cathode and a patch electrode on the chest wall was the anode. One to four shocks were delivered in these patients, with a mean of $2.1 \pm 0.8$ shocks. The cumulative stored energy of the shocks was 380 \pm 155 joules. Immediately after delivery of the shocks in these patients, the clinical VT was still inducible by programmed ventricular stimulation in 11 of 29 patients. A monomorphic VT that had not been observed clinically was induced in one patient and no VT was induced in 17 patients. During the follow-up electrophysiology test 1 week later, among the 17 patients in whom VT was noninducible immediately after delivery of the shocks, the original clinical VT was inducible in three patients, a monomorphic VT that had not been observed clinically was inducible in four patients, and no VT was inducible in ten patients. Two transseptal shocks with a mean total stored energy of $280 \pm 75$ joules were delivered in seven patients in whom the exit site of VT was in the interventricular septum. Immediately after delivery of the transseptal shocks, the clinical VT was still inducible in one of these seven patients, a monomorphic VT that had not been observed clinically was inducible in one patient, and no VT was inducible in five patients. Among the six patients in whom the clinical VT was not inducible immediately after delivery of the shocks, the follow-up electrophysiology test 1 week later demonstrated that the clinical VT was inducible in three patients, a monomorphic VT that had not been observed clinically was inducible in one patient, and no VT was inducible in two patients. 


\section{Long-Term Follow-Up}

The catheter ablation procedure was successful in 15 of 33 patients. These patients had no recurrence of symptomatic VT during a mean follow-up interval of $15.5 \pm 10$ months. An additional three patients also did not have symptomatic VT during follow-up of $28 \pm 20$ months, however these patients were treated with an antiarrhythmic drug regimen which had not been used before delivery of the shocks.

Fifteen patients experienced a recurrence of symptomatic VT during the follow-up period. Ventricular tachycardia recurred anywhere between 1 day and 8 months after attempted catheter ablation. The VT recurrence was nonfatal in nine patients and fatal in six patients (mean follow-up interval $9.8 \pm 5$ months).

The results of programmed ventricular stimulation at the 1 week follow-up electrophysiology test correlated with outcome. The follow-up electrophysiology test demonstrated no inducible VT in $67 \%$ of patients who had a successful outcome and in only $11 \%$ of patients without a successful outcome $(\mathrm{P}<0.001)$. Age, underlying heart disease, left ventricular ejection fraction, and results of mapping did not correlate with clinical outcome. Patients with an unsuccessful outcome received a greater number of shocks $(2.4 \pm 0.7$ vs 1.7 $\pm 0.6, \mathrm{P}<0.01$ ), and a greater total number of joules $(410 \pm 131$ joules vs $304 \pm 155$ joules, $\mathrm{P}$ $<0.01)$.

\section{Complications}

The mean peak creatine kinase MB fraction after the ablation procedure was $56 \pm 65 \mathrm{IU} / \mathrm{L}$ (normal range 0-10 IU/L). No patient developed new $Q$ waves on the electrocardiogram.

Five patients experienced six nonfatal complications as a result of the catheter ablation procedure. The complications consisted of sustained VT immediately postshock in one patient, ventricular fibrillation on day 5 and day 6 postshock in one patient, transient aphasia or hemiparesis in two patients, transient 2:1 atrioventricular block in one patient, atrioventricular block requiring a permanent pacemaker in one patient who received transseptal shocks, and a brachial artery thrombosis in one patient. No patients experienced a fatal complication.

\section{Discussion}

The long-term success rate of catheter ablation in preventing recurrences of symptomatic VT in the patients in this series was $45 \%$. These results indicate that catheter ablation of VT may be of value in the long-term management of selected patients who have recurrent VT. Patients in this series were selected to undergo catheter ablation for the most part only if they had one configuration of monomorphic VT that was hemodynamically stable and could be adequately mapped. Although such patients may represent a relatively small subset of patients who have recurrent VT, the catheter ablation procedure nevertheless may be extremely useful in the management of these patients if antiarrhythmic drug therapy is not effective. Many of the patients in this series were not suitable candidates for endocardial resection because of severely compromised left ventricular function, and could not be managed successfully with an antitachycardia pacemaker. In addition, an automatic implantable cardioverter/defibrillator could not be used in many patients because of frequent or incessant VT. Recurrences of VT have been prevented by catheter ablation for up to 35 months.

The catheter ablation procedure was found to be relatively safe in this series, with no fatal complications. There were no instances of myocardial perforation or cardiac tamponade and $82 \%$ of patients did not experience any complication. Complications that must be watched for include ventricular tachycardia or ventricular fibrillation, atrialventricular block, and cerebral ischemic episodes.

In each patient, an endocardial site was identified at which activation during VT preceded the onset of the QRS complexes. However, there was no significant difference between the earliest activation time in patients with and without a successful outcome. There was no absolute value for the endocardial activation time which predicted success.

In regards to the value of pace mapping, the success of the catheter ablation procedure ap- 


\section{CATHETER ABLATION OF VENTRICULAR TACHYCARDIA}

peared to be independent of whether the quality of the pace map was "excellent" or "good". It appears that concordance between pacing and VT in all 12 electrocardiographic leads is not necessary for successful ablation of VT by transcatheter shocks. However, the results of this study do not allow identification of the minimum number of concordant leads required for successful outcome.

\section{Conclusions}

Although catheter ablation of VT with intracardiac shocks clearly is capable of preventing recurrences of monomorphic VT over the longterm, its success rate is lower than the success rate of map-directed or visually directed endocardial resection. In patients with coronary artery disease who have multiple configurations of VT, endocardial resection may be appropriate if the patient has a ventricular aneurysm and particularly if there is an indication for coronary artery bypass grafting. However, in patients who are suboptimal candidates for endocardial resection because of markedly compromised left ventricular function or the absence of a ventricular aneurysm, the catheter ablation technique may provide a relatively low risk alternative to surgery and may be indicated in drug refractory patients who are not appropriate candidates for an antitachycardia pacemaker or automatic implantable cardioverter/defibrillator

Acknowledgment: The authors thank Lisa Hackbarth for her secretarial assistance. 
This document is a scanned copy of a printed document. No warranty is given about the accuracy of the copy. Users should refer to the original published version of the material. 